\title{
Investigation of the Si-rich silicon oxide by 3D atom probe tomography
}

\author{
Etienne Talbot ${ }^{1}$, Rodrigue Lardé ${ }^{1}$, Fabrice Gourbilleau ${ }^{2}$, C. Dufour ${ }^{2}$ and Philippe Pareige ${ }^{1}$ \\ ${ }^{1}$ Groupe de Physique des Matériaux, Université et INSA de Rouen, UMR CNRS 6634, avenue \\ de l'université, BP 12, 76801 Saint Etienne du Rouvray, France \\ ${ }^{2}$ Centre de Recherche sur les Ions, les Matériaux et la Photonique, équipe NIMPH, UMR CNRS \\ 6252, ENSICAEN, 6 boulevard Maréchal Juin, 14050 Caen, France
}

\begin{abstract}
Silicon rich silicon oxide multilayers for optical devices have been investigated by laser assisted wide angle atom probe tomography. Three dimensional mapping of silicon nanoclusters multilayers was obtained. The composition of the different phases were deduced and compared to theoretical concentration. These results evidenced a size distribution of the Si clusters diameter and an incomplete phase separation between silica and silicon particles.
\end{abstract}

\section{INTRODUCTION}

The miniaturization and the integration of photonic components, notably for the telecommunications, are one of the main stakes of this last decade. In particular, Si based systems presenting promising potential for realization of light sources [1, 2], waveguide amplifiers [1,3] and electroluminescence diodes compatible with the Si technology. One of the major lines deal with Silicon nanoclusters (Si-nc) embedded in silica which are promising for the realization of Si-based photonic devices. For example, Er doped silicon rich silica (SRSO) are promising candidate for planar optical amplifier at the wavelength of $1,54 \mu \mathrm{m}$, which correspond to the minimum attenuation of silica optical fiber. In these systems, Si nanoclusters (Si-nc) have a sensitizer effect on $\mathrm{Er}^{3+}$ ions, and enhanced the effective cross section of rare earth dopants by means of an efficient energy transfer between Si-nc and Er ions. To improve the properties of these devices, it will be essential to characterize more precisely Si nanoparticles and especially size distribution and interface nature between Si-nc and matrix in samples annealed in the optimized conditions of the Er emission [4,5,6].

In this paper, we report for the first time 3D mapping of Si-nc embedded in $\mathrm{SiO}_{2}$ at the atomic level. We focused our study on the local chemistry, size distribution, and Si-nc density in $\mathrm{SRSO} / \mathrm{SiO}_{2}$ multilayered structures annealed at $900^{\circ} \mathrm{C}$ during one hour. The results are compared to the "theoretical" case of complete phase separation between $\mathrm{Si}$ and $\mathrm{SiO}_{2}$.

\section{EXPERIMENT}

$\mathrm{SRSO} / \mathrm{SiO}_{2}$ multilayers (MLs) were prepared on $\mathrm{Si}$ substrates ([110] oriented) by an alternative reactive magnetron sputtering of the silica target under a plasma of $50 \% \mathrm{H}_{2}+50 \% \mathrm{Ar}$ 
to obtain SRSO sublayers, owing to the ability of hydrogen to reduce oxygen [7] and under a plasma of pure Ar to deposit the $\mathrm{SiO}_{2}$ sublayers. The MLs were grown at $650^{\circ} \mathrm{C}$ with a power density of $1.3 \mathrm{~W} . \mathrm{cm}^{-2}$ and were subsequently annealed at $900^{\circ} \mathrm{C}$ for $1 \mathrm{~h}$ under a flux of $\mathrm{N}_{2}$ mixed with $5 \%$ of $\mathrm{H}_{2}$ in order to favor the phase separation between $\mathrm{Si}$ and $\mathrm{SiO}_{2}$. The thickness of the $\mathrm{SiO}_{2}$ and SRSO sublayers are $4 \mathrm{~nm}$ and $3.8 \mathrm{~nm}$, respectively, and the size of the Si dots is limited by the thickness of the SRSO layers. High Resolution Transmission Electron Microscopy was performed using a Topcon 002B on samples prepared in the cross-section configuration.

Three-dimensional laser assisted atom probe tomography (3D-APT) analysis provides a spatial chemical map at the atomic scale of a material with an ultrahigh spatial resolution (depth resolution $\sim 0.1 \mathrm{~nm}$ ) $[8,9]$. The use of APT on semiconductor or insulator has always been limited due to the poor conductivity of these materials. Today, the recent development of the laser assisted APT allows to analyze these materials [10,11]. During the analysis, a high positive DC voltage (several kilovolts) is applied on the sample in order to create a high electric field at the apex of the tip. The superposition of femtosecond laser pulses lead to the controlled evaporation of the specimen. The evaporated ions are collected on a position-sensitive detector which is used to i) measure the time of flight of individual ions and ii) the impact position of each ion on the surface of the detector. These data permit the three dimensional reconstruction of the analyzed volume. One requirement is the sharp tip shaped sample with an end curvature radius smaller than 50nm and a small cone angle in order to create a very high electric field at the surface of the tip. The specimen is prepared using Focus Ion Beam (FIB) annular milling [12, 13]. $\mathrm{SRSO} / \mathrm{SiO}_{2}$ samples were prepared using a focused $\mathrm{Ga}$ beam $(30 \mathrm{kV})$. To prevent ion beam damage and gallium implantation, samples were protected with a $\sim 300 \mathrm{~nm}$ Cr layer and the final steps of milling were performed at low acceleration voltage $(2 \mathrm{kV})$. The results described in this paper were obtained by means of a Laser Assisted Wide Angle Tomographic Atom Probe (LAWATAP) from CAMECA. These experiments were performed at $80 \mathrm{~K}$ in an ultrahigh vacuum chamber at a pressure of $10^{-8} \mathrm{~Pa}$, and using femtosecond laser pulsing (50 $\left.\mathrm{nJ}, 350 \mathrm{fs}\right)$ at the wavelength of $343 \mathrm{~nm}$.

\section{DISCUSSION}

The cross-sectional HRTEM image of the $\mathrm{SRSO} / \mathrm{SiO}_{2}$ multilayers is shown on Figure 1.(a). A careful observation of this micrograph allows to reveal the presence of crystallized $\mathrm{Si}$ nanoclusters. Si-nc are only localized in the SRSO sublayers. As expected by the multilayers structures, no clusters with a diameter greater than the thickness of the SRSO layers are detected. However, due to the poor contrast between silica matrix and Si-nc, small or amorphous nanoclusters are not distinguishable with such a technique. Therefore, it is difficult to estimate the density and the size distribution of the Si-nc. The nominal composition, stacking of all MLs, was $\mathrm{X}_{\mathrm{Si}}=41 \%$ and $\mathrm{X}_{\mathrm{O}}=59 \%$. This corresponds to an excess of silicon of $\sim 11 \%$ for the MLs, which is about $25 \%$ in the SRSO sublayers. Under the hypothesis of complete phase separation between $\mathrm{Si}$ and $\mathrm{SiO}_{2}\left(\mathrm{SiO}_{x} \rightarrow \frac{x}{2} \mathrm{SiO}_{2}+\left(1-\frac{x}{2}\right) \mathrm{Si}\right)$, we can calculate the particles density in the MLs. In this case, all Si-nc have a diameter of $3.8 \mathrm{~nm}$, a sharp interface with the matrix and the density of Si-nc is about $\sim 5.1 \times 10^{18} \mathrm{Si}-\mathrm{nc} / \mathrm{cm}^{3}$. A schematic representation of this case is shown on Figure 1.(b). 


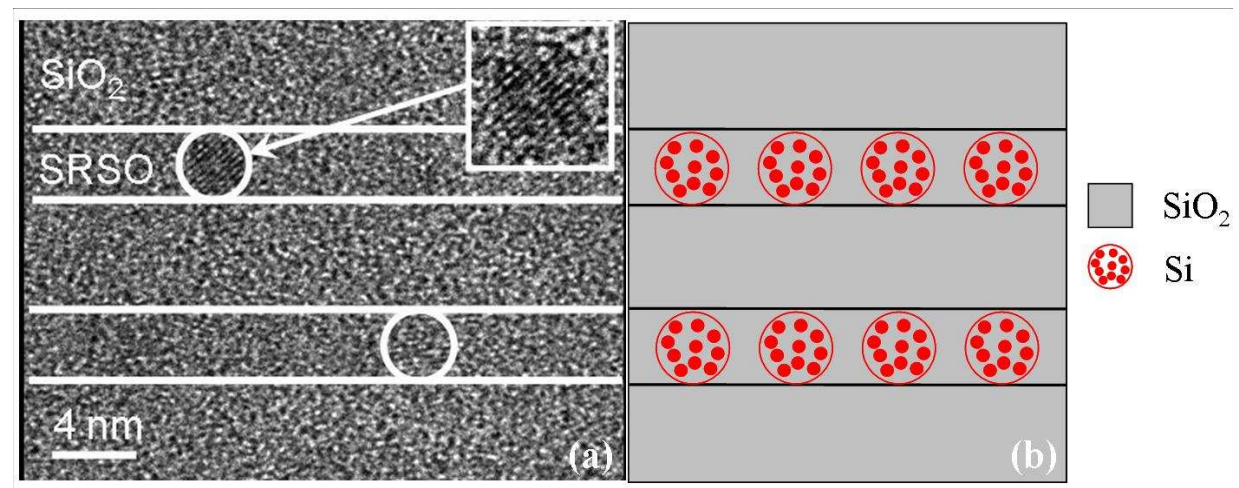

Figure 1 : (a) High-Resolution TEM image of the SRSO (3.8nm) / $\mathrm{SiO}_{2}$ (4nm) multilayers. Crystallized silicon nanoclusters can be seen (circle and magnified image); (b) Schematic drawing of the MLs configuration in the case of a complete phase separation between Si and $\mathrm{SiO}_{2}$ phases.

\section{D-APT results}

We have performed Laser Assisted Atom Probe Tomography analyses on these $\mathrm{SRSO} / \mathrm{SiO}_{2}$ MLs. A typical 3D reconstruction of the analysed volume is shown in Figure 2.(a). Alternating silica and silicon rich silica (indicate by arrows) regions are clearly distinguishable on this image. These results clearly evidence the alternating composition of the sample with layer of pure silica and layer of Si-nc in silica matrix. The associated concentration profile along the sample growth direction is presented in Figure 2.(b). The abscise axis corresponds to the ML's growth direction. The overall measured composition is $\mathrm{X}_{\mathrm{Si}} \approx 43.0 \pm 0.3 \%$ and $\mathrm{X}_{\mathrm{O}} \approx 57.0 \pm 0.3 \%$, which correspond to a $\mathrm{Si}$ excess of $\sim 14 \%$, in good agreement with the nominal composition. The concentration in sublayers of pure silica is $\mathrm{X}_{\mathrm{Si}} \approx 34.3 \pm 0.3 \%$ and $\mathrm{X}_{\mathrm{O}} \approx 65.7 \pm 0.3 \%$, and it is $\mathrm{X}_{\mathrm{Si}}$ $\approx 51.0 \pm 0.3 \%$ and $\mathrm{X}_{\mathrm{O}} \approx 49.0 \pm 0.3 \%$ in the SRSO sublayers. The Si excess reached in the SRSO regions is about $\sim 26 \%$ in good agreement with the nominal content. 

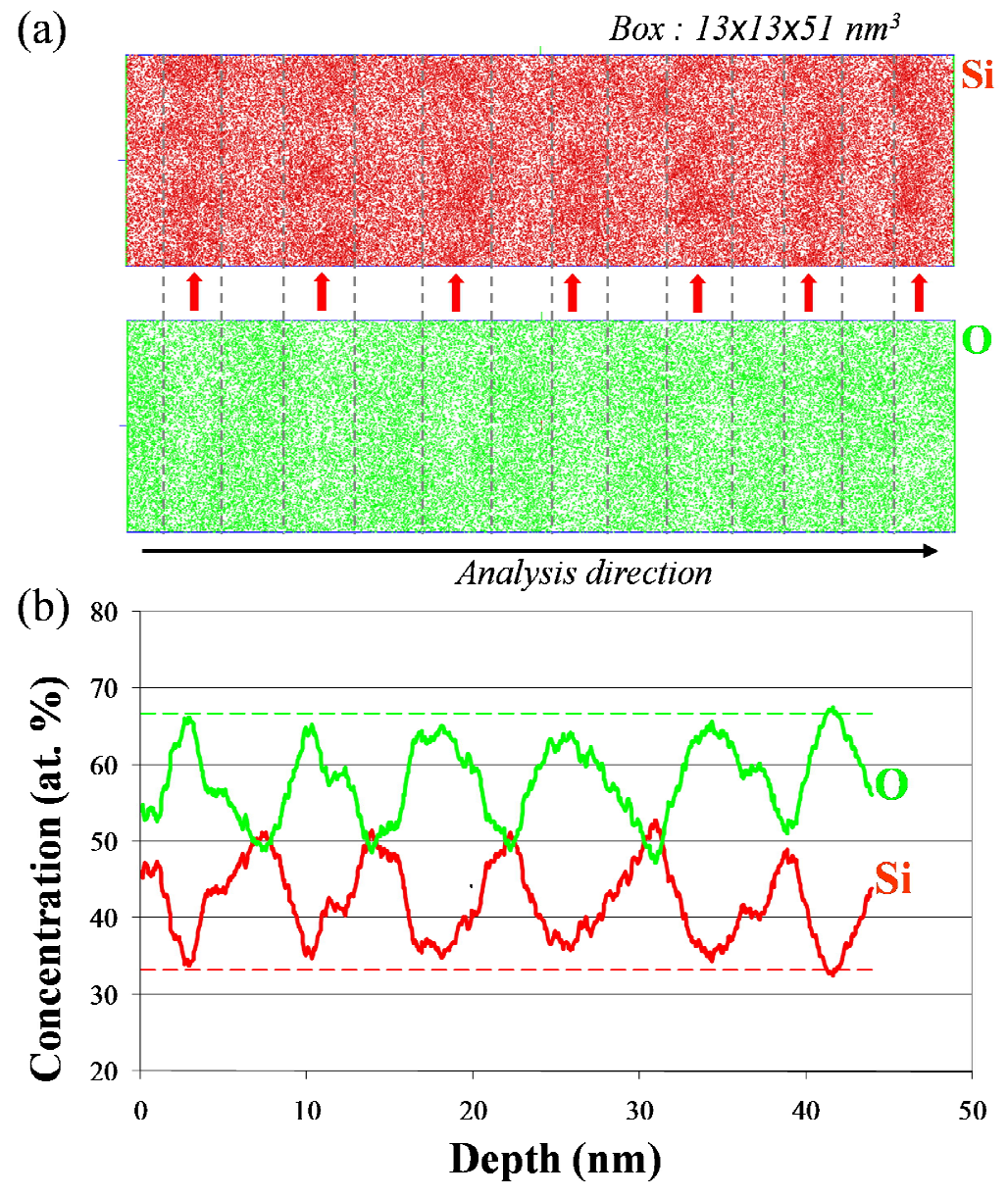

Figure 2 : (a) 3D reconstruction of ${\mathrm{SRSO} / \mathrm{SiO}_{2}}_{2} \mathrm{ML}$ 's analysed by LA-WATAP (arrows and dashed-line indicate rich Si regions). The volume is $13 \times 13 \times 51 \mathrm{~nm}^{3}$. (b) Composition profile along the multilayer.

Figure 3.(a) shows a 3D iso-concentration surface image of the multilayers which reveals the presence of Si-nc (red-zones) embedded in silica regions (green-zones). This image clearly evidenced the expected uniform distribution of Si-nc in the SRSO sublayers alternatively with pure silica sublayers after a $1 \mathrm{~h}$-annealing at $900^{\circ} \mathrm{C}$. Data treatment of the $3 \mathrm{D}$-APT analyses allow us to investigate independently, at the atomic scale, each nano-cluster or the matrix (excluding Si-nc). First, the composition of the SRSO matrix (measured after subtraction of the Si-nc cluster), which corresponds to the decomposition of the surrounding Si-nc, has been obtained. The measured SRSO matrix composition is $\mathrm{X}_{\mathrm{Si}}{ }^{\text {matrix }} \approx 41.9 \pm 0.3 \%$ and $\mathrm{X}_{\mathrm{O}}{ }^{\text {matrix }} \approx 58.1 \pm 0.3 \%$, which is significantly higher than pure silica phase $\left(\mathrm{X}_{\mathrm{Si}}=33.3 \%\right.$ and $\left.\mathrm{X}_{\mathrm{O}}=66.7 \%\right)$ and indicating an incomplete phase separation. The Si excess remaining in the SRSO sublayers after $1 \mathrm{~h}-$ annealing at $900^{\circ} \mathrm{C}$ is $\sim 13 \%$, i.e. only $\sim 50 \%$ of the $\mathrm{Si}$ excess has precipitated. The annealing conditions $\left(1 \mathrm{~h}\right.$ at $900^{\circ} \mathrm{C}$ ) are not long enough to ensure a complete phase separation of the system. These results confirm the slow phase separation process at this temperature as suggested by Iacona et al. [14]. 

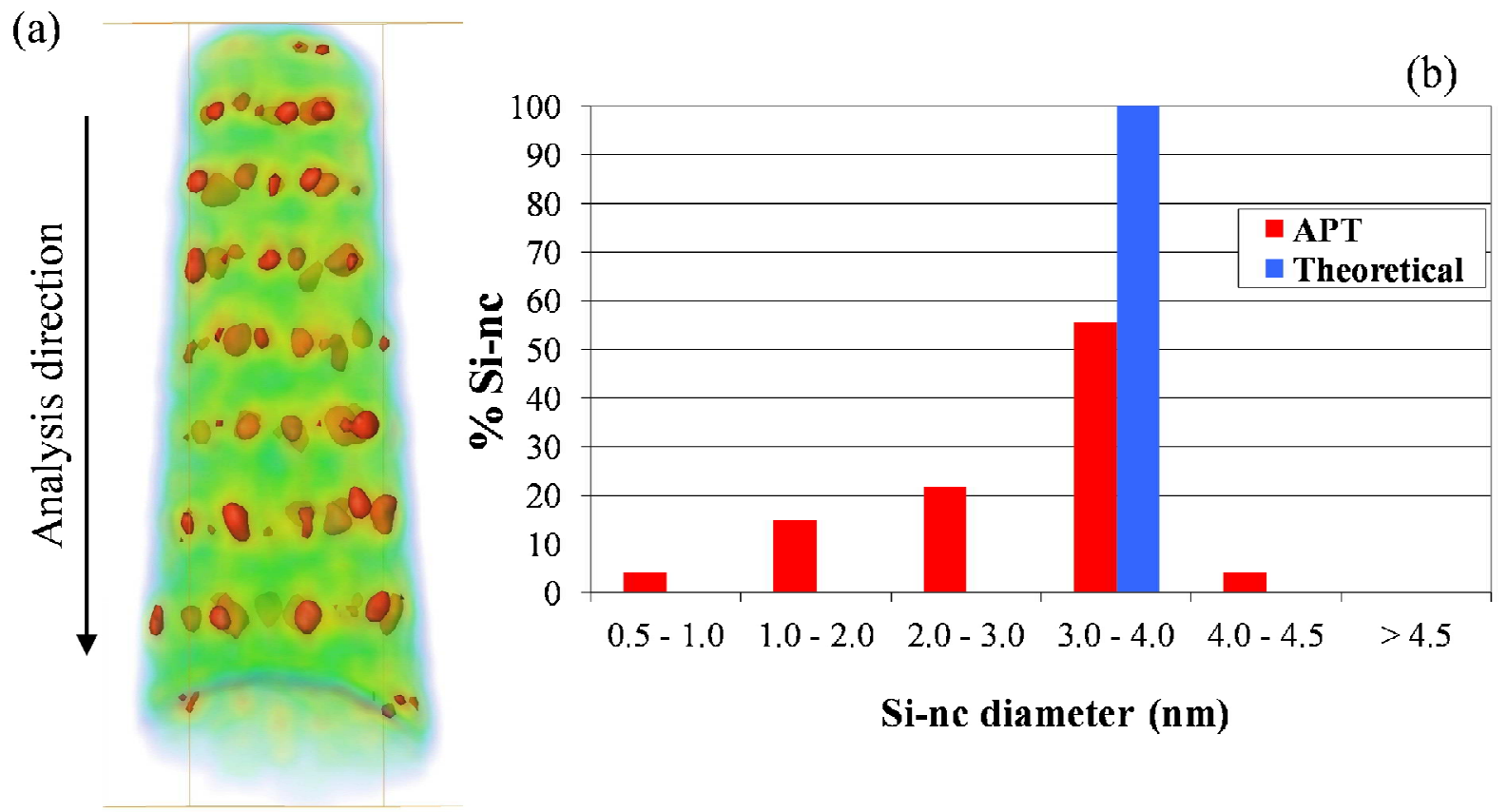

Box : $25 \times 25 \times 70 \mathrm{~nm} 3$

Si-nc diameter $(\mathrm{nm})$

Figure 3 : (a) 3D iso-concentration of the SRSO/SiO2 ML's (red zones indicate Si-nc and green zones indicate silica). (b) Measured and theoretical size distribution of the Si-nc diameter in the SRSO layers.

Finally, the Si-nc size distribution in the $\mathrm{SRSO} / \mathrm{SiO}_{2}$ multilayers was studied and compared to the ideal case of a complete phase separation. An accurate size distribution of the silicon clusters in the SRSO layers is presented in figure 3.(b). The detections of small Si-nc embedded in silica matrix by APT highlight the capability of this technique compared to other ones (HRTEM, XRD, Raman spectroscopy, EFTEM ...). On the figure 3.(b), the diameter ranges from 0.5 to $4.5 \mathrm{~nm}$. The mean diameter was estimated to $2.9 \mathrm{~nm}$. The silicon rich silicon oxide - silica multilayers was used to prevent $\mathrm{Si}$ diffusion in the silica during phase separation and the growth of clusters, and as a consequence limit the Si-nc size to the thickness of the SRSO sublayers. As expected, the presence of the $\mathrm{SiO}_{2}$ sublayers on each side of the SRSO one limits efficiently the diameter of the Si-nc which has been found in majority to be less than $3.8 \mathrm{~nm}$ (i.e. less than the thickness of the SRSO layer). As evidenced by the size distribution (figure 3.b), there are small clusters in the ML's. Their presence can be explained by the incomplete phase separation between $\mathrm{Si}$ and $\mathrm{SiO}_{2}$. The detection of $\mathrm{Si}$ particles with a diameter greater than silica sublayers thickness can be partially explained by a poor interface quality between $\mathrm{SiO}_{2}$ and $\mathrm{SRSO}$ layers and uncertainties on the layer thickness. The Si nanocluster number density is estimated to $5.7 \pm 1.0 \times 10^{18} \mathrm{Si}$ $\mathrm{nc} / \mathrm{cm}^{3}$. This result can be compared to the "theoretical" prediction of a complete phase separation between $\mathrm{Si}$ and $\mathrm{SiO}_{2}$ phases considering that all nanoclusters have a diameter of 3.8 $\mathrm{nm}$ (figure 3.b). In this case, the Si-nc density would be about $5.1 \times 10^{18} \mathrm{Si}-\mathrm{nc} / \mathrm{cm}^{3}$. The measured density is in good agreement with the hypothesis of a complete phase separation, but our size distribution evidenced the fact that annealing time and/or annealing temperature are not so long to favour homogeneous Si-nc size density. 


\section{CONCLUSIONS}

In summary, $\mathrm{Si}$ nanoclusters array in $\mathrm{SRSO} / \mathrm{SiO}_{2}$ multilayered structures have been investigated by an original approach by means of three-dimensional atom probe tomography. 3D-APT results show at the atomic scale the characteristic of the structure. In the annealing conditions investigated here, i.e. $900^{\circ} \mathrm{C}$, our results show an incomplete phase separation between $\mathrm{Si}$ and $\mathrm{SiO}_{2}$. This is evidenced by a $\mathrm{Si}$ supersaturation in the SRSO layers and a large scale distribution size of the Si-nc.

\section{ACKNOWLEDGMENTS}

This work was supported by the Upper Normandy Region and the French Ministry of Research in the framework of Research Networks of Upper-Normandy.

\section{REFERENCES}

1. L. Pavesi, L. Dal Negro, C. Mazzoleni, G. Franzo and F. Priolo, Nature, 408, 440 (2000).

2. L. Canham, Appl. Phys. Lett., 57, 1046 (1990).

3. L. T. Canham, Nature, 408, 411 (2000).

4. F. Gourbilleau, M. Levalois, C. Dufour, J. Vicens and R. Rizk, J. Appl. Phys., 95, 3717 (2004).

5. M. Wodjak, M. Klik, M. Forcales, O.B. Gusev, T. Gregorkiewicz, D. Pacifici, G. Franzo, F. Priolo and F. Iacona, Phys. Rev. B, 69, 233315 (2004).

6. G. Franzo, S. Boninelli, D. Pacifici, F. Priolo F. Iacona, and C. Bongiorno, Appl. Phys. Lett., 82, 3871 (2003).

7. F. Gourbilleau, X. Portier, C. Ternon, P. Voivenel, R. Madelon and R. Rizk, Appl. Phys. Lett., 78, 3058 (2001).

8. D. Blavette, A. Bostel, J. M. Sarrau, B. Deconihout and A. Menand, Nature, 363, 432 (1993).

9. M. K. Miller, Atom Probe Tomogrpahy: Analysis at the Atomic Level, Springer 2000.

10. G. Kellog and T. Tsong, J. Appl. Phys., 51, 1184 (1980).

11. B. Gault, F. Vurpillot, M. Gilbert, A. Vella, A. Menand, D. Blavette and B. Deconihout, Rev. Sci. Instrum., 77, 043705 (2006).

12. G. B. Thomson, M. K. Miller and H. L. Fraser, Ultramicroscopy, 100, 25 (2004).

13. D. J. Larson, D. R. Foord, A. K. Perford-Long, H. Liew, M. G. Blamire, A. Cerezo, and G.

D. W. Smith, Ultramicroscopy, 79, 287 (1999).

14. F. Iacona, C. Bongiorno, C. Spinella, S. Boninelli and F. Priolo, J. Appl. Phys., 95, 3723

(2004). 